\title{
Recovering an Homogeneous Polynomial from Moments of Its Level Set
}

\author{
Jean B. Lasserre
}

Received: 25 May 2012 / Revised: 21 June 2013 / Accepted: 12 August 2013 /

Published online: 27 August 2013

(C) Springer Science+Business Media New York 2013

\begin{abstract}
Let $\mathbf{K}:=\{\mathbf{x}: g(\mathbf{x}) \leq 1\}$ be the compact (and not necessarily convex) sublevel set of some homogeneous polynomial $g$. Assume that the only knowledge about $\mathbf{K}$ is the degree of $g$ as well as the moments of the Lebesgue measure on $\mathbf{K}$ up to order $2 d$. Then the vector of coefficients of $g$ is the solution of a simple linear system whose associated matrix is nonsingular. In other words, the moments up to order $2 d$ of the Lebesgue measure on $\mathbf{K}$ encode all information on the homogeneous polynomial $g$ that defines $\mathbf{K}$ (in fact, only moments of order $d$ and $2 d$ are needed).
\end{abstract}

Keywords Homogeneous polynomials $\cdot$ Sublevel sets $\cdot$ Moments $\cdot$ Inverse problem from moments

\section{Introduction}

The inverse problem of reconstructing a geometrical object $\mathbf{K} \subset \mathbb{R}^{n}$ from the only knowledge of moments of some measure $\mu$ whose support is $\mathbf{K}$ is a fundamental problem in both applied and pure mathematics with important applications in e.g. computer tomography, inverse potentials, signal processing, and statistics and probability, to cite a few. In computer tomography, for instance, the X-ray images of an object can be used to estimate the moments of the underlying mass distribution, from which one seeks to recover the shape of the object that appears on some given images. In gravimetry applications, the measurements of the gravitational field can be converted into information concerning the moments, from which one seeks to recover the shape of the source of the anomaly.

J. B. Lasserre $(\bowtie)$

LAAS-CNRS and Institute of Mathematics, University of Toulouse LAAS, 7 avenue du Colonel Roche, BP 54200, 31031 Toulouse Cedex 4, France

e-mail: lasserre@1aas.fr 
Of course, exact reconstruction of objects $\mathbf{K} \subset \mathbb{R}^{n}$ is in general impossible unless $\mathbf{K}$ has very specific properties. For instance, if $\mathbf{K}$ is a convex polytope then exact recovery of all its vertices has been shown to be possible via a variant of what is known as Prony method. Only a rough bound on the number of vertices is required and relatively few moments suffice for exact recovery. For more details the interested reader is referred to the recent contribution of Gravin et al. [3] and the references therein. On the other hand, Cuyt et al. [1] have shown that approximate recovery of a general $n$-dimensional shape is possible by using an interesting property of multidimensional Padé approximants, analogous to the Fourier slice theorem for the Radon transform.

\section{Contribution}

From previous contributions and their references, it is transparent that exact recovery of an $n$-dimensional shape is a difficult problem that can be solved only in a few cases. Therefore identifying such cases is of theoretical and practical interest. The goal of this paper is to identify one such case as we show that exact recovery is possible when $\mathbf{K} \subset \mathbb{R}^{n}$ is the compact and not necessarily convex sublevel set $\left\{\mathbf{x} \in \mathbb{R}^{n}: g(\mathbf{x}) \leq 1\right\}$ associated with an homogeneous polynomial $g$. By exact recovery we mean recovery of all coefficients of the polynomial $g$. In fact, exact recovery is not only possible but rather straightforward as it suffices to solve a linear system with a nonsingular matrix! Moreover, only moments of order $d$ and $2 d$ of the Lebesgue measure on $\mathbf{K}$ are needed. As already mentioned, exact recovery is possible only if $\mathbf{K}$ has very specific properties and indeed, crucial in the proof is a property of levels sets associated with homogeneous polynomials (and in fact, also true for level sets of positively homogeneous nonnegative functions).

\section{Main Result}

\subsection{Notation and Definitions}

Let $\mathbb{R}[\mathbf{x}]$ be the ring of polynomials in the variables $\mathbf{x}=\left(x_{1}, \ldots, x_{n}\right)$ and let $\mathbb{R}[\mathbf{x}]_{d}$ be the vector space of polynomials of degree at most $d$ (whose dimension is $s(d):=\left(\begin{array}{c}n+d \\ n\end{array}\right)$ ). For every $d \in \mathbb{N}$, let $\mathbb{N}_{d}^{n}:=\left\{\alpha \in \mathbb{N}^{n}:|\alpha|\left(=\sum_{i} \alpha_{i}\right)=d\right\}$, and let $\mathbf{v}_{d}(\mathbf{x})=\left(\mathbf{x}^{\alpha}\right), \alpha \in \mathbb{N}^{n}$, be the vector of monomials of the canonical basis $\left(\mathbf{x}^{\alpha}\right)$ of $\mathbb{R}[\mathbf{x}]_{d}$. Denote by $\mathcal{S}_{k}$ the space of $k \times k$ real symmetric matrices with scalar product $\langle\mathbf{B}, \mathbf{C}\rangle=\operatorname{trace}(\mathbf{B C})$; also, the notation $\mathbf{B} \succeq 0$ (resp. $\mathbf{B} \succ 0$ ) stands for $\mathbf{B}$ is positive semidefinite (resp. positive definite). Let $\ell(\bar{d}):=\left(\begin{array}{c}n+d-1 \\ d\end{array}\right)$.

A polynomial $f \in \mathbb{R}[\mathbf{x}]_{d}$ is written

$$
\mathbf{x} \mapsto f(\mathbf{x})=\sum_{\alpha \in \mathbb{N} n} f_{\alpha} \mathbf{x}^{\alpha}
$$

for some vector of coefficients $\mathbf{f}=\left(f_{\alpha}\right) \in \mathbb{R}^{s(d)}$. 
A real-valued polynomial $g: \mathbb{R}^{n} \rightarrow \mathbb{R}$ is homogeneous of degree $d(d \in \mathbb{N})$ if $g(\lambda \mathbf{x})=\lambda^{d} g(\mathbf{x})$ for all $\lambda$ and all $\mathbf{x} \in \mathbb{R}$. Given $g \in \mathbb{R}[\mathbf{x}]$, denote by $G \subset \mathbb{R}^{n}$ the sublevel set $\{\mathbf{x}: g(\mathbf{x}) \leq 1\}$.

If $g$ is homogeneous then $G$ is compact only if $g$ is nonnegative on $\mathbb{R}^{n}$ (and so $d$ is even). Indeed suppose that $g\left(\mathbf{x}_{0}\right)<0$ for some $\mathbf{x}_{0} \in \mathbb{R}^{n}$; then by homogeneity, $g\left(\lambda \mathbf{x}_{0}\right)<0$ for all $\lambda>0$ and so $G$ contains a half-line and cannot be compact.

\subsection{Main Result}

The main result is based on the following result of independent interest valid for positively homogeneous functions (and not only homogeneous polynomials). A function $f: \mathbb{R}^{n} \rightarrow \mathbb{R}$ is positively homogeneous of degree $d \in \mathbb{R}$ if $f(\lambda \mathbf{x})=\lambda^{d} f(\mathbf{x})$ for all $\lambda>0$ and all $\mathbf{x} \in \mathbb{R}^{n}$.

Lemma 1 Let $f: \mathbb{R}^{n} \rightarrow \mathbb{R}$ be a measurable, positively homogeneous and nonnegative function of degree $0<d \in \mathbb{R}$, with bounded level set $\{\mathbf{x}: f(\mathbf{x}) \leq 1\}$. Then for every $k \in \mathbb{N}$ and $\alpha \in \mathbb{N}^{n}$ :

$$
\int_{\{\mathbf{x}: f(\mathbf{x}) \leq 1\}} \mathbf{x}^{\alpha} f(\mathbf{x})^{k} \mathrm{~d} \mathbf{x}=\frac{n+|\alpha|}{n+k d+|\alpha|} \int_{\{\mathbf{x}: f(\mathbf{x}) \leq 1\}} \mathbf{x}^{\alpha} \mathrm{d} \mathbf{x} .
$$

Proof To prove (2.1) we use an argument already used in [4,5]. With $\alpha \in \mathbb{N}^{n}$, let $\tilde{\alpha}:=\left(\alpha_{2}, \ldots, \alpha_{n}\right) \in \mathbb{N}^{n-1}$ and define $\mathbf{z}:=\left(z_{2}, \ldots, z_{n}\right)$.

Let $\phi: \mathbb{R}_{+} \rightarrow \mathbb{R}$ be measurable and consider the integral $\int_{\mathbb{R}^{n}} \phi(g(\mathbf{x})) \mathbf{x}^{\alpha} \mathrm{d} \mathbf{x}$. Using the change of variable $x_{1}=t$ and $x_{i}=t z_{i}$ for all $i=2, \ldots, n$, and invoking homogeneity, one obtains:

$$
\begin{aligned}
\int_{\mathbb{R}^{n}} \phi(f(\mathbf{x})) \mathbf{x}^{\alpha} \mathrm{d} \mathbf{x} & =\int_{\mathbb{R}^{n}} \phi\left(t^{d} f\left(1, z_{2}, \ldots, z_{n}\right)\right) t^{n+|\alpha|-1} \mathbf{z}^{\tilde{\alpha}} \mathrm{d}(t, \mathbf{z}) \\
& =d^{-1}\left(\int_{0}^{\infty} u^{(n+|\alpha|) / d-1} \phi(u) \mathrm{d} u\right) \times A_{\alpha} \\
\text { with } A_{\alpha} & =\int_{\mathbb{R}^{n-1}} \mathbf{z}^{-\tilde{\alpha}} f(1, \mathbf{z})^{-(n+|\alpha|) / d} \mathrm{~d} \mathbf{z} .
\end{aligned}
$$

Hence the choices $t \mapsto \phi(t):=\mathrm{I}_{[0,1]}(t)$ and $t \mapsto \phi(t):=t^{k} \mathrm{I}_{[0,1]}(t)$ yield

$$
d \int_{\{\mathbf{x}: f(\mathbf{x}) \leq 1\}} \mathbf{x}^{\alpha} \mathrm{d} \mathbf{x}=A_{\alpha} \int_{0}^{1} u^{(n+|\alpha|) / d-1} \mathrm{~d} u=\frac{A_{\alpha} d}{n+|\alpha|}
$$




$$
d \int_{\{\mathbf{x}: f(\mathbf{x}) \leq 1\}} f(\mathbf{x})^{k} \mathbf{x}^{\alpha} \mathrm{d} \mathbf{x}=A_{\alpha} \int_{0}^{1} u^{(n+k d+|\alpha|) / d-1} \mathrm{~d} u=\frac{A_{\alpha} d}{n+k d+|\alpha|},
$$

respectively. And so (2.1) follows.

With $g \in \mathbb{R}[\mathbf{x}]_{d}$ being an homogeneous polynomial of degree $d$, consider now the matrix $\mathbf{M}_{d}(\lambda)$ of moments of order $2 d$ associated with the Lebesgue measure on $G=\{\mathbf{x}: g(\mathbf{x}) \leq 1\}$, that is, $\mathbf{M}_{d}(\lambda)$ is a real square matrix with rows and columns indexed by the monomials $\mathbf{x}^{\alpha}, \alpha \in \mathbb{N}_{d}^{n}$, and with entries

$$
\mathbf{M}_{d}(\lambda)[\alpha, \beta]=\int_{\{\mathbf{x}: g(\mathbf{x}) \leq 1\}} \mathbf{x}^{\alpha+\beta} \mathrm{d} \mathbf{x}=: \lambda_{\alpha+\beta} \quad \forall \alpha, \beta \in \mathbb{N}_{d}^{n}
$$

So all entries of $\mathbf{M}_{d}(\lambda)$ are moments of order $2 d$. Our main result is as follows:

Theorem 2 Let $g \in \mathbb{R}[\mathbf{x}]_{d}$ be homogeneous of degree $d$ with unknown coefficient vector $\mathbf{g} \in \mathbb{R}^{\ell(d)}$ and with compact level set $G=\{\mathbf{x}: g(\mathbf{x}) \leq 1\}$. Assume that one knows the moments $\lambda=\left(\lambda_{\alpha}\right)$ for the Lebesgue measure on $G$, for every $\alpha \in \mathbb{N}^{n}$ with $|\alpha|=2 d$ and $|\alpha|=d$. Then

$$
\mathbf{g}=\frac{n+d}{n+2 d} \mathbf{M}_{d}(\lambda)^{-1} \lambda^{(d)},
$$

where $\lambda^{(d)}=\left(\lambda_{\alpha}\right), \alpha \in \mathbb{N}_{d}^{n}$, is the vector of all moments of order $d$.

Proof Use (2.1) with $k=1$ and $|\alpha|=d$ to obtain

$$
\sum_{\beta \in \mathbb{N}^{n}:|\beta|=2 d} g_{\beta} \lambda_{\alpha+\beta}=\frac{n+d}{n+2 d} \lambda_{\alpha} \quad \forall|\alpha|=d,
$$

or in matrix form

$$
\mathbf{M}_{d}(\lambda) \mathbf{g}=\frac{n+d}{n+2 d} \lambda_{d}
$$

from which the desired result follows if $\mathbf{M}_{d}(\lambda)$ is non singular. But this follows from the fact that $G$ has nonempty interior. Indeed if $\mathbf{M}_{d}(\lambda) \mathbf{u}=0$ for some nontrivial vector $\mathbf{u} \in \mathbb{R}^{\ell(d)}$ (with $\ell(d)=\left(\begin{array}{c}n+d-1 \\ d\end{array}\right)$ ) then denoting by $u \in \mathbb{R}[\mathbf{x}]_{d}$ the homogeneous polynomial with coefficient vector $\mathbf{u}$, one obtains the contradiction

$$
0=\left\langle\mathbf{u}, \mathbf{M}_{d}(\lambda) \mathbf{u}\right\rangle=\int_{G} u(\mathbf{x})^{2} \lambda(\mathrm{d} \mathbf{x}) \neq 0 .
$$



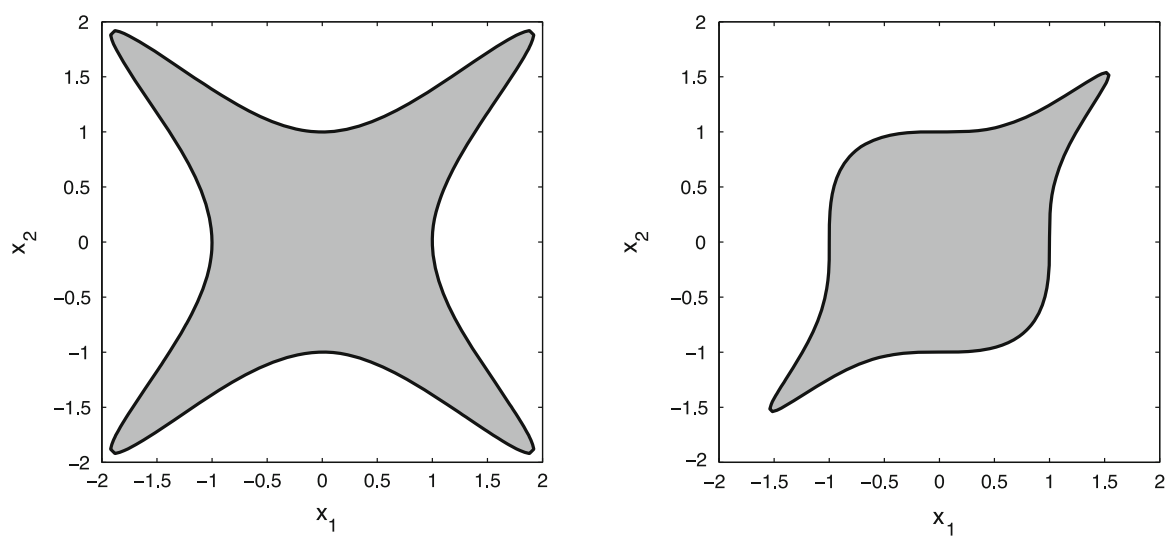

Fig. $1 G$ with $x_{1}^{4}+x_{2}^{4}-1.925 x_{1}^{2} x_{2}^{2}$ and $x_{1}^{6}+x_{2}^{6}-1.925 x_{1}^{3} x_{2}^{3}$

There are alternative ways for obtaining $\mathbf{g}$ from the moments $\lambda$. It suffices to apply (2.1) for a family $\mathcal{F}$ of multi-indices $\alpha \in \mathbb{N}^{n}$ whose cardinality $|\mathcal{F}|$ matches the dimension $\left(\begin{array}{c}n+d-1 \\ d\end{array}\right)$ of the vector $\mathbf{g}$.

For instance, with $n=2$ and $d=2$ ( $g$ is a quadratic form with vector of coefficients $\left.\mathbf{g}=\left(g_{20}, g_{11}, g_{02}\right)\right), \mathbf{g}$ can also be obtained by

$$
\mathbf{g}=\left[\begin{array}{lll}
\lambda_{20} & \lambda_{11} & \lambda_{02} \\
\lambda_{30} & \lambda_{21} & \lambda_{12} \\
\lambda_{21} & \lambda_{12} & \lambda_{30}
\end{array}\right]^{-1}\left[\begin{array}{l}
\frac{n}{n+2} \lambda_{00} \\
\frac{n+1}{n+3} \lambda_{10} \\
\frac{n+1}{n+3} \lambda_{01}
\end{array}\right],
$$

provided that the above inverse matrix exists.

Similarly, with $n=2$ and $d=4$ ( $g$ is a quartic form with vector of coefficients $\left.\mathbf{g}=\left(g_{40}, g_{31}, g_{22}, g_{13}, g_{04}\right)\right), \mathbf{g}$ can also be obtained by

$$
\mathbf{g}=\left[\begin{array}{lllll}
\lambda_{40} & \lambda_{31} & \lambda_{22} & \lambda_{13} & \lambda_{04} \\
\lambda_{50} & \lambda_{41} & \lambda_{32} & \lambda_{23} & \lambda_{14} \\
\lambda_{41} & \lambda_{32} & \lambda_{23} & \lambda_{14} & \lambda_{05} \\
\lambda_{60} & \lambda_{51} & \lambda_{42} & \lambda_{33} & \lambda_{24} \\
\lambda_{42} & \lambda_{33} & \lambda_{24} & \lambda_{15} & \lambda_{06}
\end{array}\right]^{-1}\left[\begin{array}{l}
\frac{n}{n+4} \lambda_{00} \\
\frac{n+1}{n+5} \lambda_{10} \\
\frac{n+1}{n+5} \lambda_{01} \\
\frac{n+2}{n+6} \lambda_{20} \\
\frac{n+2}{n+6} \lambda_{02}
\end{array}\right],
$$

provided that the above inverse matrix exists.

It is worth emphasizing that the sub-level set $G$ is not necessarily convex. For instance, Fig. 1 displays $G$ for $g(\mathbf{x})=x_{1}^{4}+x_{2}^{4}-1.925 x_{1}^{2} x_{2}^{2}$ and $g(\mathbf{x})=x_{1}^{6}+x_{2}^{6}-$ $1.925 x_{1}^{3} x_{2}^{3}$.

\section{Concluding Remark}

It is worth emphasizing that in the above approach the problem is still quite overdetermined as one requires more than $\left(\begin{array}{c}n+d-1 \\ d\end{array}\right)$ moments to recover the coefficients of the 
homogeneous polynomial $g$. This is in contrast to the so-called quadrature domains in the plane. For instance, the 3 vertices (hence 6 parameters) of an arbitrary triangle $T \subset \mathbb{R}^{2}$ are uniquely determined from the 3 harmonic (complex) moments $\int_{T}\left(x_{1}+i x_{2}\right)^{k} \mathrm{~d} \mathbf{x}, k=1,2,3$. In fact $2 n$ such harmonic moments are required to recover all vertices of $n$-gons; see e.g. [2, pp. 1224-1225]. So for sub-level sets of homogeneous polynomials, an issue of further investigation is to determine (or provide an upper bound on) the minimum number of moments required to recover the coefficients of the homogeneous polynomial.

\section{References}

1. Cuyt, A., Golub, G., Milanfar, P., Verdonk, B.: Multidimensional integral inversion, with applications in shape reconstruction. SIAM J. Sci. Comput. 27(3), 1058-1070 (2005)

2. Golub, G.H., Milanfar, P., Varah, J.: A stable numerical method for inverting shape from moments. SIAM J. Sci. Comput. 21(4), 1222-1243 (1999)

3. Gravin, N., Lasserre, J.B., Pasechnik, D.V., Robins, S.: The inverse moment problem for convex polytopes. Discrete Comput. Geom. 48(3), 596-621 (2012)

4. Morosov, A., Shakirov, S.: New and old results in resultant theory. Theor. Math. Phys. 163(2), 587 (2010). http://connection.ebscohost.com/c/articles/51280373/new-old-results-resultant-theory

5. Morosov, A., Shakirov, S.: Introduction to integral discriminants. J. High Energy Phys. 12, 002 (2009). http://iopscience.iop.org/1126-6708/2009/12/002 